Research Paper

\title{
Higher Dosage of HIFU Treatment May Lead to Higher and Longer Efficacy for Moderate to Severe Perennial Allergic Rhinitis
}

Hongquan Wei ${ }^{1 凶}$, Yong Zhang ${ }^{2}$, Lei Shi ${ }^{1}$, Jingru Zhang1, Yan Xia ${ }^{1}$, Jian Zang ${ }^{1}$, Aihui Yan ${ }^{1}$, Wei $\mathrm{Li}^{1}$ and Xuejun Jiang ${ }^{1}$

1. Department of Otolaryngology, The First Hospital of China Medical University, Shenyang, Liaoning 110001, P.R. China

2. Department of Pathology, College of Basic Medical Sciences, China Medical University. Shenyang, Liaoning 110001, P.R. China

$\square$ Corresponding author: Hongquan Wei, MD. Ph.D. Department of Otolaryngology, The First Hospital of China Medical University, 155 North Nanjing Street, Shenyang, Liaoning 110001, P.R. China. Email: hongquanwei@163.com, Phone: 86-13940437387

(c) Ivyspring International Publisher. This is an open-access article distributed under the terms of the Creative Commons License (http://creativecommons.org/ licenses/by-nc-nd/3.0/). Reproduction is permitted for personal, noncommercial use, provided that the article is in whole, unmodified, and properly cited.

Received: 2013.07.08; Accepted: 2013.II.II; Published: 2013.11.27

\begin{abstract}
Objectives: This study was to compare the efficacies and side effects of high intensity focused ultrasound (HIFU) treatment for perennial allergic rhinitis (PAR) with regular and increased dosage.

Study design: A prospectively assembled cohort was retrospectively analyzed through visual analogue scale (VAS).

Methods: Regular dosage of HIFU treatment was applied to 56 PAR patients in group A. An increased dosage as twice as the regular one was applied to 48 patients in group B. Nasal obstruction, sneezing, rhinorrhea and rhinocnesmus, which were recognized as the four main symptoms of allergic rhinitis (AR), were evaluated before treatment, 3 months after treatment, and I year after treatment. The satisfaction of patients was also evaluated at I year postoperatively. Biopsy of the inferior turbinate and morphometric analysis were applied to II patients in group $A$ and 10 in group B before HIFU treatment and 3 months after treatment.
\end{abstract}

Results: Comparing the AR symptoms before treatment, There is no statistical difference observed between group $A$ and $B(p>0.05)$. The four main symptoms at 3 months and I year after treatment were all significantly improved $(p<0.0 I)$ in both group $A$ and $B$. The VAS scores of $A R$ symptoms in Group B were lower than those in Group A at the same stage after treatment, especially at I year after treatment $(p<0.05)$. Comparing the results at 3 months and I year after treatment, a tendency of recurrence of these symptoms was observed statistically in group $A$ $(p<0.05)$, but not in group $B(p>0.05)$. More cases of nasal dryness and perirhinal swelling were found in group $B$ than those in group $A(p<0.05)$, while all side effects were mild and temporary. Patients in group $B$ were more satisfied than those in group $A(p=0.0866>0.05)$, though not statistically significant. More reduction of the eosinophils, other inflammatory cells, and the submucosal glands was observed after HIFU treatment in group $B$ than that in group $A(p<0.05)$.

Conclusions: A proper increment of HIFU dosage may be recommended to meet the needs of more improvement of AR symptoms and less recurrence.

Key words: high intensity focused ultrasound (HIFU); perennial allergic rhinitis (PAR); surgical treatment

\section{Introduction}

As their names are so called, ultrasonic or supersonic waves are mechanical vibrations in solid, liquid, or gaseous mediums, with the frequencies ly- ing above the range of human hearing. Piezo-electric effects are adapted to the generation of ultrasound at frequencies above $500 \mathrm{kc}$., which is the range most 
often used in biological work. In 1942, Lynn et al. designed, built and successfully operated an efficient generator of focused ultrasound[1]. The high intensity focused ultrasound (HIFU) could produce focal heating in the centers of paraffin blocks, and destruct focal areas deep in fresh liver tissue with minimal effects at the surface and no effects on the intervening tissue.

Surgical resection has been a standard treatment in selected cases with solid tumors. However, due to the tumor sites, advanced stages, or poor general conditions, many patients can't undergo surgeries. HIFU is a novel non-invasive technique that is able to produce coagulative necrosis at an accurate focal point within the tissue, without damaging overlying and adjacent structures even within the path of an ultrasonic wave[2]. The thermal ablation of tumor with HIFU has been applied to thousands of patients with prostate tumors[3,4], uterine fibroids[5], liver tumors[6], pancreatic cancer[7], bone tumors[8] and renal tumors[9] worldwide over the last ten years.

Ultrasound imaging-guided HIFU (USgHIFU) system [Chingqing Haifu (HIFU) Tech Co., Ltd., Chongqing, China] was first equipped in Asia, then in Europe. In the United States, the Food and Drug Administration (FDA) approved the magnetic resonance guided focused ultrasound surgery (MRgFUS) for clinical treatments of uterine fibroids in 2004. HIFU was demonstrated to be safe, effective and feasible in treating human solid tumors[2]. Several years ago, a CZB-HIFU therapeutic instrument for allergic rhinitis (AR) was developed by the same company and first equipped in China. Currently, this instrument has been applied to patients with moderate to severe perennial allergic rhinitis (PAR). Some patients resistant to drug treatments and those who could not or would not accept nasal spray and oral administration were also considered for HIFU treatment in clinical practice.

A safe dosage of HIFU for AR treatment was recommended for practice. This safe and maybe low dosage led to the following results: less effect and recurring earlier compared with other treatments [10]. We gradually increased the dosage until it was twice the amount of the original one. By dividing the patients into two groups, a group treated by the original dosage and the other group treated by the increased dosage, their curative effects, side effects and satisfaction results were compared.

\section{Patients and methods}

\section{Clinical data}

104 patients enrolled in this study met the diagnosis of moderate to severe PAR described in Allergic
Rhinitis and its Impact on Asthma (ARIA) in 2008[11]. All patients came from the Department of Otolaryngology, the First Hospital of China Medical University. 56 of them were divided into group A, who had been given HIFU treatment of regular dosage from July, 2009 to December, 2010. The other 48 of them were divided into group $B$, who had been given HIFU treatment for twice the time of the regular dosage from January to December, 2011. Among patients in group A, 29 were male, and 27 were female. The average age was 35 years (range from 12 to 63). Their AR history ranged from 1 to 27 years with an average of 7.37 years. 31 patients in group A were given general anesthesia, and 25 patients selected topical anesthesia. Among those in group B, 26 were male, and 22 were female. The average age was 32 years (range from 11 to 58). Their AR history ranged from 3 to 21 years with an average of 6.98 years. The patients in group B were all given general anesthesia. All patients in both group A and group B had been given drug treatment before enrollment but didn't get satisfactory therapeutic effect. Patients with any of the following were excluded: a. Pregnancy or lactation; b. nasal polyps, sinusitis, invert papilloma and other neoplasms; c. onset period of asthma or infection of upper respiratory tract; $\mathrm{d}$. previous nose surgery; e. immunological treatments in 3 months, or other clinical treatment in 1 month; $f$. treatment of long acting corticosteroid in 1 month, or treatment of other antihistamine, corticosteroid, anticholinergic, $\beta$-receptor agonist or sedatives in 2 weeks; g. other severe diseases of heart, lung, kidney, blood or neural system which might be infeasible to accept HIFU treatment.

The study protocol was approved by the Institutional Review Board of the First Hospital of China Medical University. Informed consents were obtained from all patients.

\section{Methods}

All patients were put in supine position. Topical anesthesia was applied on bilateral mucosa of the nasal cavity with cotton slivers which had been dipped into $2 \%$ dicaine and $1 \%$ adrenaline. This procedure was repeated three times for 3-5 minutes at each time. When all sites requiring treatment were anesthetized sufficiently, CZB-HIFU therapeutic instrument (Chongqing HIFU Technology Co.Ltd.) for rhinitis was applied at gear III as the power parameter. The emitter window of ultrasound was put against the lateral wall of nasal cavity anterior and superior to agger nasi, the inferior turbinate and corresponding nasal septum, then scanned between front and back, then up and down. The scan speed on lateral wall was $2 \mathrm{~mm} / \mathrm{sec}$, and $4 \mathrm{~mm} / \mathrm{sec}$ on nasal septum. For patients in group $\mathrm{A}$, the unilateral treatment 
time on lateral wall was 200 seconds and 50 seconds on nasal septum. The total treatment time for both sides was 500 seconds. The treatment power was 1645 w. For patients in group B, the unilateral treatment time on lateral wall was 400 seconds and 100 seconds on nasal septum. The gross time on both sides was 1000 seconds while the power was 3290 w. Tamponade of nasal cavity, and administered medication were not necessary.

\section{Evaluation of effect and satisfaction}

A retrospective review was performed to all patients who underwent HIFU treatment. Surveys were conducted to evaluate symptoms using a visual analog scale grading system before operation, 3 months after operation, and 1year after operation. Nasal obstruction, sneezing, rhinorrhea, and rhinocnesmus were graded by patients using a system scale from zero to ten. Zero referred to no symptoms at all while ten represented the most severe situation which could be imagined by patients. At the final of postoperative survey, patients were all required to self-evaluate their satisfaction of HIFU treatment from zero (completely unsatisfied) to ten (completely satisfied). According to the satisfaction scores, we categorized the satisfaction as excellent $(\geq 9$ and $\leq 10)$, very good ( $\geq$ 7 and $<9)$, good $(\geq 5$ and $<7)$, poor $(\geq 3$ and $<5)$, and very poor $(\geq 0$ and $<3)$.

Furthermore, patients were also asked if they had experienced any postoperative complications. If the answer was "yes", they would be asked more about the severity and duration of the symptoms, and whether any additional treatment was required to relieve these symptoms.

\section{Biopsy and Morphometric Analysis}

Before HIFU treatment and 3 months after treatment, biopsy specimens of the inferior turbinate were collected from 11 patients in group A and 10 patients in group B. Three sections of each specimen were $\mathrm{HE}$ stained and observed at the magnification of $200 \times$. A high power field with epithelium was randomly selected from each section. The eosinophils, other inflammatory cells and submucosal glands were counted.

\section{Statistical Methods}

Paired $t$ test was applied to compare the VAS scores of each symptom before and after treatment within the same group. Kruskal-Wallis and Mann-Whitney tests were applied to compare indexes between two groups. Chi-square test was applied to compare the side effect incidence and satisfaction rates between the two groups. A $p$ value $<0.05$ was regarded as statistical significance. All tests were done with IBM SPSS Statistic Version 20.

\section{Results}

\section{Patients were not completely satisfied with topical anesthesia}

In group A, 25 patients were given topical anesthesia. 11 of them experienced pain from mild to middle level. 3 of them felt itching and sneezed many times during operation. A young female patient who finally dropped out of this study could not even endure the pain until that we adjusted the power parameter from gear III to gear II. The other 31 patients in group A and all in group B selected general anesthesia.

\section{Patients in group B presented more significant and longer effect of HIFU treatment than those in group $A$}

In both $\mathrm{A}$ and $\mathrm{B}$ groups, all symptoms (nasal obstruction, sneezing, rhinorrhea and rhinocnesmus) related to AR were improved after treatment. No matter 3 months or 1 year after treatment, all VAS scores of symptoms were significantly lower than those before treatment $(p<0.01)$.

Comparing group $\mathrm{A}$ and $\mathrm{B}$, the pre-operation VAS scores of same AR symptom were at the same level. There was no statistical difference observed $(p>0.05) .3$ months after treatment, there was still no difference in the evaluations of sneezing and rhinorrhea $(p>0.05)$, while better results relating to nasal obstruction and rhinocnesmus were shown in group B than those in group A $(p<0.05)$. At 1 year after treatment, evaluations of all AR symptoms in group B were better than those in group A $(p<0.05)$.

In group $\mathrm{A}$, the VAS scores at 1year were higher than those at 3 months after treatment $(p<0.05)$, which suggested all symptoms had partially come back. In group B, there was no difference observed in any of the four AR symptoms $(p>0.05)$, which means the treatment effects were well kept after a year in this group. (Table 1)

\section{More mild and temporary side effects were shown in group $B$ than in group $A$}

Following treatment, almost all patients in both groups temporarily suffered obvious nasal obstruction caused by swelling mucosa and jelly-like discharge. Mild rhinocnesmus and sneezing might be combined. 7 to 10 days later, these symptoms were markedly relieved automatically in both group A and B. Beside the above side effects, nasal dryness happened in $7.14 \%(4 / 56)$ of patients in group $\mathrm{A}$, and relieved within 3 months. The incidence in group $B$ was $20.83 \%(10 / 48)$, which is statistically higher than that in group A $(p<0.05)$. Most of these cases $(9 / 10)$ 
relieved within 5 months. One patient with the longest history of nasal dryness recovered after 8 months. Furthermore, perirhinal swelling was observed in $12.50 \%(7 / 56)$ of patients in group $\mathrm{A}$, which disappeared in 7 to 10 days (average 8 days). In group B, the incidence of perirhinal swelling was $89.58 \%$ $(43 / 48)$, which was significantly higher than that in group A $(p<0.01)$. The swelling cases were all spontaneously healed in 8 to 14 days (average 12 days) (Figure 1). Additional treatment was not necessary for all of above side effects.

Nasal bleeding, synechia, perforation of nasal septum, numbness, headache, or visual disturbance was not found in group A or B.
The evaluation of satisfaction in group $B$ is higher than that in group $A$ though not statistically significant

The VAS score of satisfaction in group B was higher than that in group A $(8.38 \pm 1.51$ vs $7.46 \pm 2.57)$, though not statistically significant $(p=0.123>0.05)$ (Figure 2). More patients in group B than those in group A were categorized as "excellent" $[54.17 \%(26 / 48)$ vs $44.64 \%(25 / 56)]$ and "very good" [27.08\%(13/48) vs $16.07 \%(9 / 56)]$. Less in group B than that in group A was $\operatorname{good}[18.75 \%(9 / 48)$ vs. $28.58 \%(16 / 56)]$, poor[0\%(0/48) vs. $7.14 \%(4 / 56)]$, or very poor $[0 \%(0 / 48)$ vs. $3.57 \%(2 / 56)]$. Patients in group $\mathrm{B}$ were more satisfied than those in group A $(p=0.0866>0.05)$. (Figure 3$)$

Table I. VAS evaluation of Nasal Symptoms

\begin{tabular}{lllll}
\hline Symptoms & groups & Pre-operation & 3 months after treatment & 1 year after treatment \\
\hline Nasal obstruction & A & $8.46 \pm 2.49||$ & $3.07 \pm 1.91^{*} \S$ & $3.57 \pm 2.56^{*}+\S$ \\
Sneezing & B & $8.56 \pm 1.99||$ & $2.13 \pm 1.18^{*} \S$ & $2.25 \pm 1.54^{*} \neq \S$ \\
& A & $7.43 \pm 2.11||$ & $3.18 \pm 2.62^{*}||$ & $3.64 \pm 2.59^{*}+\S$ \\
Rhinorrhea & B & $7.56 \pm 2.99||$ & $2.19 \pm 1.76^{*}||$ & $2.23 \pm 1.75^{*} \neq \S$ \\
& A & $7.50 \pm 2.25||$ & $3.39 \pm 2.97^{*}||$ & $3.79 \pm 2.93^{*}+\S$ \\
Rhinocnesmus & B & $7.44 \pm 2.95||$ & $2.19 \pm 1.61^{*}||$ & $2.31 \pm 1.85^{*} \neq \S$ \\
& A & $5.43 \pm 3.15||$ & $2.71 \pm 2.69^{*} \S$ & $3.11 \pm 2.67^{*}+\S$ \\
\hline
\end{tabular}

Abbreviations: VAS, visual analog scale

Compared with the preoperative VAS score of the same group, $\mathrm{P}<0.01^{*}$

Compared with the VAS score of the same group at 3 months after treatment, $\mathrm{P}<0.05 \dagger$, or $\mathrm{P}>0.05 \ddagger$

Paired t-test was applied for above statistical analysis $(\alpha=0.05)$

Compared with the VAS score of the other group at the same stage of treatment, $\mathrm{P}<0.05 \S$, or $\mathrm{P}>0.05$ |

Kruskal-Wallis Test was applied for the analysis $(\alpha=0.05)$

\section{Side Effects after Treatment}

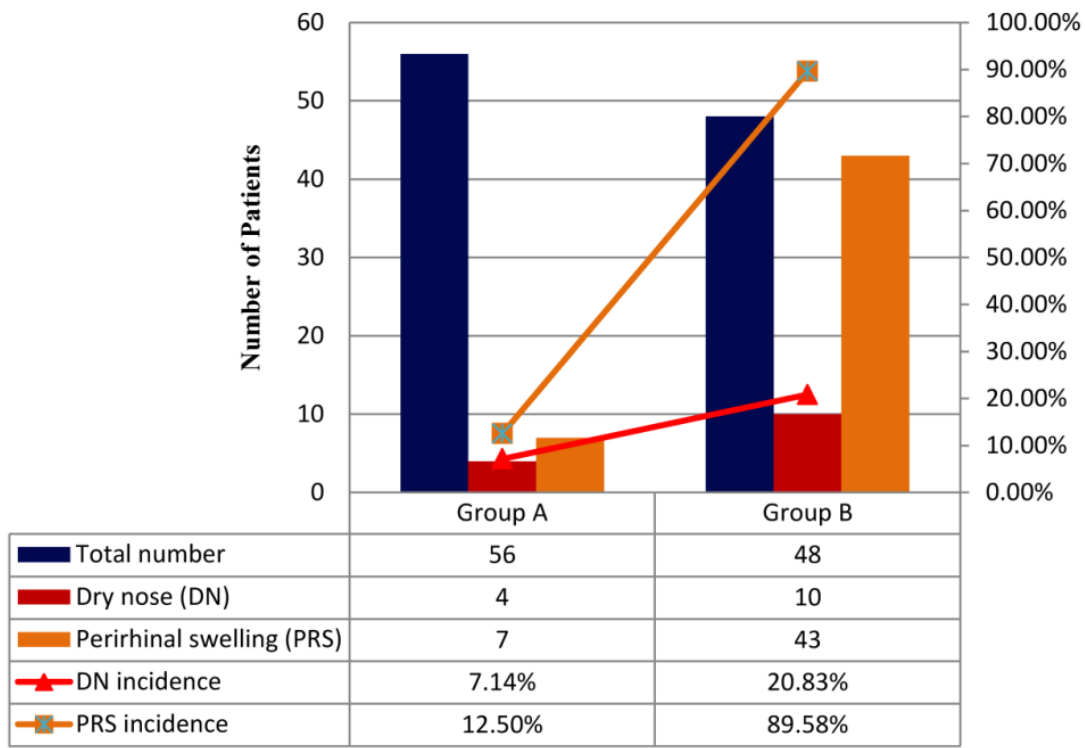

Figure I. The incidence of dry nose of group B (20.83\%) was significantly higher than that of group $A(7.14 \%)$. $x^{2}=4.1584, P<0.05$. The incidence of perirhinal swelling of group B (89.5\%) was obviously higher than that of group $A(12.50 \%) . x^{2}=6 I .5205, P<0.0$ I. Chi-square test was applied for statistical analysis $(\alpha=0.05)$ 
VAS Evaluation of Satisfaction

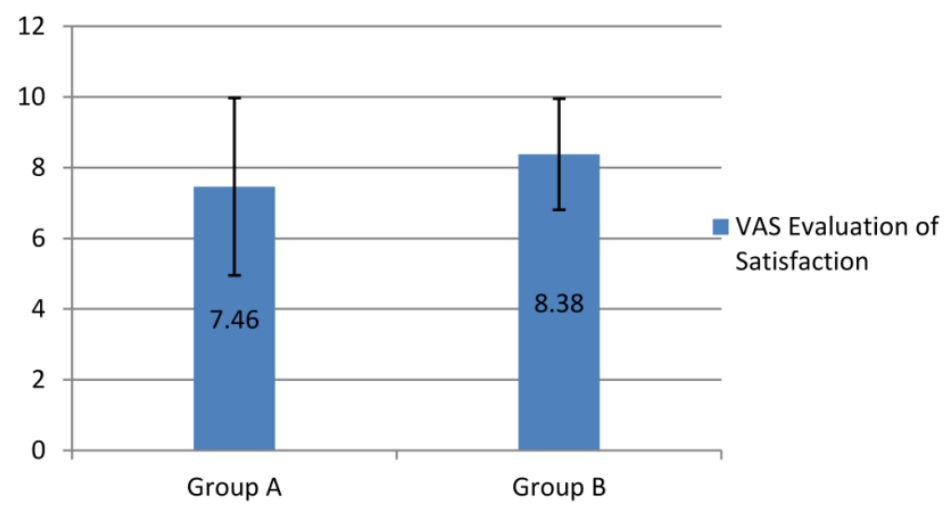

Figure 2. The VAS score of satisfaction in group $B(8.38 \pm I .5 \mathrm{I})$ was higher than that in group $A(7.46 \pm 2.57)$, though not statistically significant $(P=0.123$, $P>0.05$ ). Mann-Whitney Test (two-tailed) was applied for analysis.

\section{Categorization of the VAS Evaluation of} Satisfaction

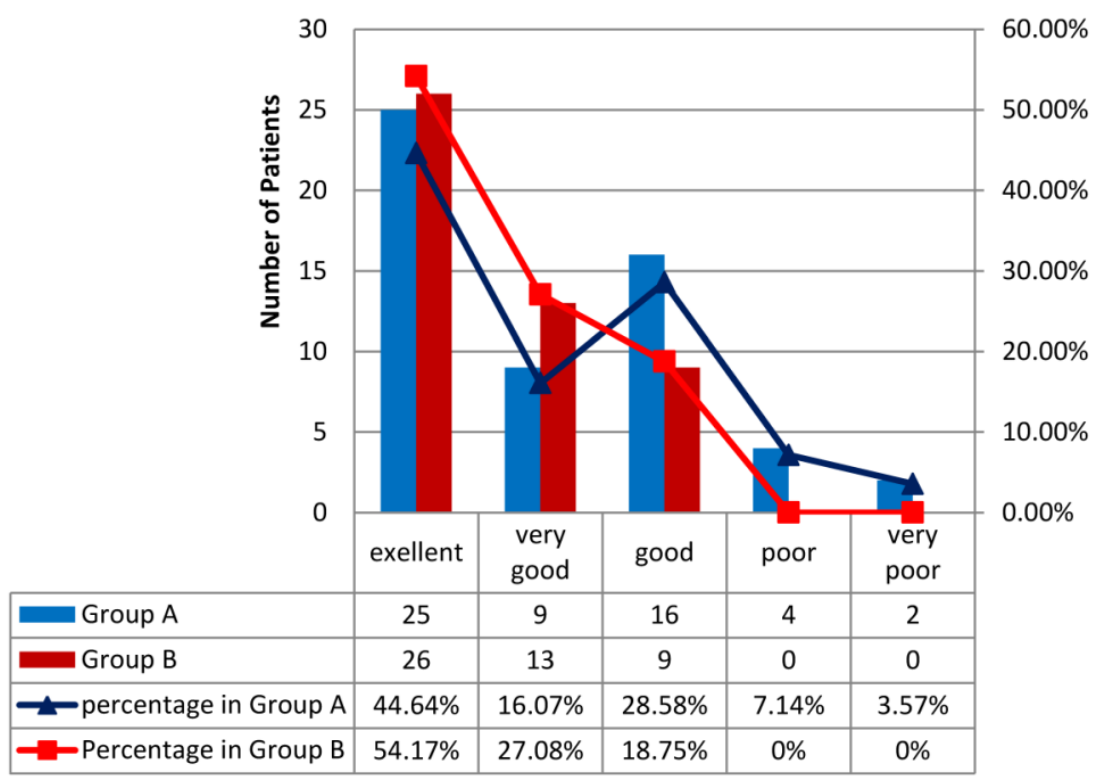

Figure 3. More patients in group B than those in group A were categorized as "excellent" and "very good". Less in group B than that in group A was good, poor, or very poor. Patients in group $B$ were more satisfied that those in group $A(p=0.0866>0.05)$, though not statistically significant. Chi-square test was applied for statistical analysis $(\alpha=0.05)$.

\section{The eosinophils, other inflammatory cells and the submucosal glands were more significantly decreased in group $B$ than that in group $A$}

Obvious interstitial edema, abundant submucosal glands and a lot of inflammatory cells could be observed in the nasal turbinate mucosa of PAR patients. It was changed after HIFU treatment (Figure 4). Morphometric analysis showed that the eosinophils, other inflammatory cells, and the submucosal glands were all decreased at 3 months after HIFU treatment in both group A and B $(p<0.001)$. It was more significant in group B (Table 2).

\section{Discussion}

HIFU treatment for AR has been applied in China for several years. Topical anesthesia was chosen for most cases. Under the power gear III, the total treatment time usually ranged from 360 to $600 \mathrm{sec}$ $[10,12]$. In this study, we chose $500 \mathrm{sec}$ as the total treatment time in group $\mathrm{A}$ and double treatment time in group B. However, the patients were not completely satisfied with topical anesthesia in this study. Irritation of mucosa of nasal cavity might induce the attack of itch and sneezing. Insufficient numbness caused pain at different levels. According to our observation, general anesthesia is recommended. 


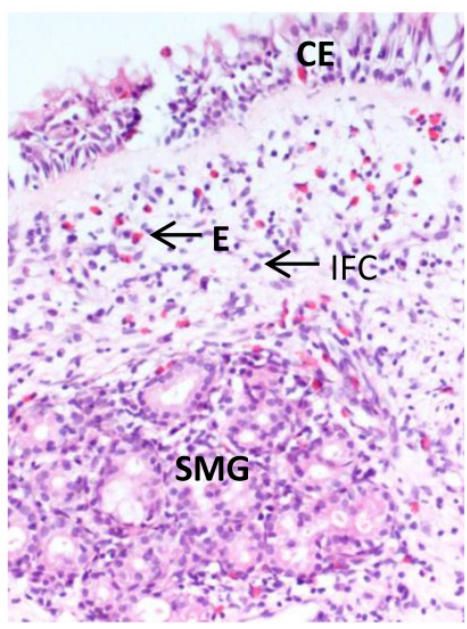

A

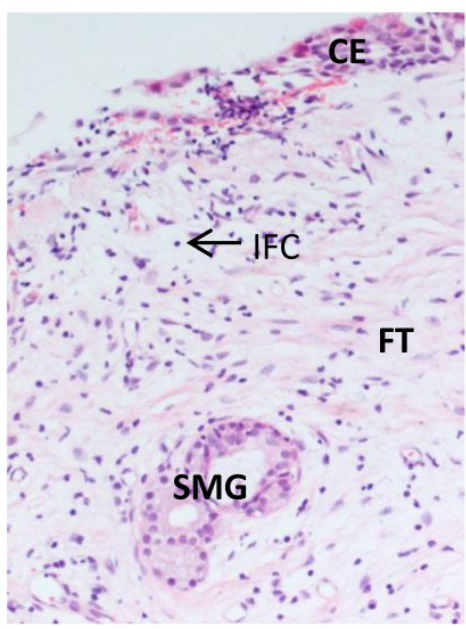

B

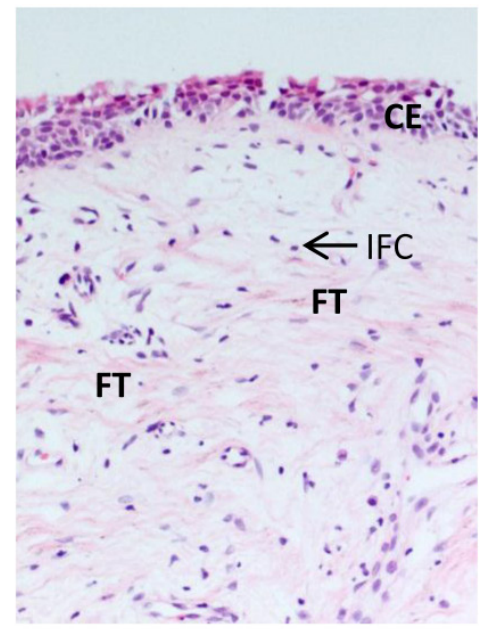

C

Figure 4. Histology of the inferior turbinate mucosa before and after the HIFU treatment. Compared to the nasal mucosa of the PAR patients before treatment $(A)$, the ciliated epithelium is well preserved and the interstitial edema is alleviated after HIFU treatment in group $A(B)$. The eosinophils, other inflammatory cells, and the submucosal glands are reduced while more fibrous tissues are present after treatment. These changes are more significant in the specimens obtained from group B (C). CE: ciliated epithelium; E: eosinophil; FT: fibrous tissues; IFC: other inflammatory cells; SMG: submucosal glands.

Table 2. Morphometric analysis of the inferior turbinate specimens before and after HIFU treatment

\begin{tabular}{lllll}
\hline & Group & Before HIFU treatment & 3 months after HIFU treatment & $p$-Value \\
\hline Eosinophils $\left(/ \mathrm{mm}^{2}\right)$ & A & $81.6 \pm 64.9^{*}$ & $45.7 \pm 30.4 \dagger$ & $p<0.0001$ \\
& B & $77.3 \pm 59.6^{*}$ & $32.6 \pm 25.2 \dagger$ & $p<0.0001$ \\
Other inflammatory & A & $104.9 \pm 51.8^{*}$ & $69.6 \pm 35.5 \ddagger$ & $p<0.0001$ \\
cells $\left(/ \mathrm{mm}^{2}\right)$ & B & $98.6 \pm 58.3^{*}$ & $50.1 \pm 38.7 \ddagger$ & $p<0.0001$ \\
Submucosal glands $\left(/ \mathrm{mm}^{2}\right)$ & A & $11.2 \pm 4.8^{*}$ & $5.3 \pm 3.9 \dagger$ & $p<0.0001$ \\
& B & $12.5 \pm 4.3^{*}$ & $3.9 \pm 2.8 \dagger$ & $p<0.0001$ \\
\hline
\end{tabular}

Compared to the results before HIFU treatment, the densities of the eosinopils, other inflammatory cells, and the submucosal glands of the inferior turbinate mucosa are all very significantly decreased after treatment in both group A and $B(p<0.0001)$. Paired t-test was applied $(\alpha=0.05)$.

Comparing the densities of the cells and glands between group A and B, there is no significant difference observed before treatment $\left(p>0.05^{*}\right)$. After HIFU treatment, the differences of the eosinophils and submucosal glands are significant $(\mathrm{p}<0.05 \mathrm{t})$, and that of other inflammatory cells is highly significant between group A and B ( $\mathrm{p}<0.01 \ddagger)$. Kruskal-Wallis Test was applied for the analysis $(\alpha=0.05)$

Comparing the VAS scores before and after HIFU treatment, AR symptoms were improved in group A and were more improved in group B. Before treatment, the VAS scores of the AR symptoms in group A and B were at the same level $(p>0.05)$. Either 3 months or 1 year after treatment, the VAS score of four AR symptoms in group B were all lower than those in group A. These differences were statistically significant except those of sneezing and rhinorrhea at 3 months after treatment. $10.71 \%(6 / 56)$ of patients in group A were not satisfied (VAS score of satisfaction $<5$ ) and gave "poor" or "very poor" evaluation. Inefficiency or recurrence was their most frequent complaint. No patient in group B had the same complaint or was not satisfied. Moreover, statistical differences were observed between VAS scores at 3 months and 1 year after treatment in group A $(p<0.05)$, but not in group B $(p>0.05)$. These results proposed that AR symptoms were best controlled at 3 months after
HIFU treatment, and recurred partially after 1 year in group A but not in group B. Higher dosage of HIFU treatment results in better and longer curative effects.

On the other side, higher dosage of HIFU treatment caused higher incidence of side effects. The incidences of nasal dryness and perirhinal swelling in Group B were significantly higher than those in group A $(p<0.01)$. The power of HIFU is focused on the submucosal layer of the nasal cavity. In the focal spot, the tissue in a small volume is heated rapidly. Higher temperatures cause tissue boiling and bubble formation[13,14]. Subsequent tissue coagulation and necrosis may directly destruct glands in the submucosal layer and decrease their secretion. The occlusion of blood vessels and thrombus formation reduce the exudation from the plasma. The above changes contribute to relief of mucosal swelling and watery discharge, as well as leading to temporary nose dryness in some cases. The perirhinal swelling was related to 
the treatment of the innervation area of the anterior ethmoidal nerve. The anterior ethmoidal nerve provides sensory branches to the anterior and superior part of the nasal cavity. It comes from the nasociliary nerve, and gives rise to internal and external nasal branches. Passing the bony margin of piriform aperture, the external branch of the anterior ethmoidal nerve ultimately innervates skin on the lateral side of the nose. As a heating effect of HIFU treatment, edema happened around the pathway of the anterior ethmoidal nerve and its branches. Higher dosage of treatment caused more exudation from the submucosal layer inside the nasal cavity to the subcutaneous layer around the nose.

Vidian neurectomy[15], turbinectomy, electrocautery, cryosurgery, chemosurgery[16], microwave, laser[17] and radiofrequency[18] are most commonly-used surgical treatments for AR. These treatments are proved to be effective while dry eyes, nasal bleeding, crusting, and synechia are often found postoperatively. None of the above side effects was observed in group A or B after HIFU treatment.

In this study, the eosinophils, other inflammatory cells, and the submucosal glands were all decreased in both group A and B at 3 months after HIFU treatment. Compared to group $\mathrm{A}$, the reduction was more significant in group B. It partially explained the better and longer efficacy of the HIFU treatment with higher dosage through pathological study. Vessels and nerves in deeper layers of the inferior turbinate should be changed similarly. Further study might be needed.

At 1 year after HIFU treatment, the self-evaluation of satisfaction in group B was better than that in group A, though not statistically significant $(p=0.0866>0.05)$. A further study with more cases was suggested. Compared with the significant improvement of AR symptoms, the satisfaction of patients in group $B$ might have been decreased by the side effects with higher incidences and longer duration.

\section{Conclusion}

As a time consuming procedure, HIFU treatment has to be applied with an ideal time to balance the improvements of AR symptoms and side effects. HIFU treatment with a properly increased dosage produces more improvements of AR symptoms, longer curative effects and induces higher incidences of mild and temporary side effects at the same time. It may be considered to meet the needs of more improvement and less recurrence, when reasonable risk of side effects could be accepted.

\section{Acknowledgements}

We are grateful to Mr. Stephen Crano and Mr. Dan Barlow for their kind helps to polish this manuscript. This study was supported by General Grant (L2010617) of Education Department of Liaoning Province, Science and Technology Design Program (2011225027) of Science and Technology Agency of Liaoning Province, China.

\section{Competing Interests}

The authors have declared that no competing interest exists.

\section{References}

1. Lynn JG, Zwemer RL, Chick AJ, Miller AE. A New Method for the Generation and Use of Focused Ultrasound in Experimental Biology. The Journal of general physiology 1942; 26:179-193.

2. Orsi F, Arnone P, Chen W, Zhang L. High intensity focused ultrasound ablation: a new therapeutic option for solid tumors. Journal of cancer research and therapeutics 2010; 6:414-420.

3. Madersbacher S, Pedevilla M, Vingers L, Susani M, Marberger M. Effect of high-intensity focused ultrasound on human prostate cancer in vivo. Cancer research 1995; 55:3346-3351.

4. Napoli A, Anzidei M, De Nunzio Cet al Real-time Magnetic Resonance-guided High-intensity Focused Ultrasound Focal Therapy for Localised Prostate Cancer: Preliminary Experience. European urology 2013; 63:395-398.

5. Park MI, Kim YS, Keserci B, Rhim H, Lim HK. Volumetric MR-guided high-intensity focused ultrasound ablation of uterine fibroids: treatment speed and factors influencing speed. European radiology 2012.

6. Wijlemans JW, Bartels LW, Deckers Ret al. Magnetic resonance-guided high-intensity focused ultrasound (MR-HIFU) ablation of liver tumours. Cancer imaging : the official publication of the International Cancer Imaging Society 2012; 12:387-394.

7. Khokhlova TD, Hwang JH. HIFU for palliative treatment of pancreatic cancer. Journal of gastrointestinal oncology 2011; 2:175-184.

8. Li C, Zhang W, Fan W, Huang J, Zhang F, Wu P. Noninvasive treatment of malignant bone tumors using high-intensity focused ultrasound. Cancer 2010; 116:3934-3942

9. Klingler HC, Susani M, Seip R, Mauermann J, Sanghvi N, Marberger MJ. A novel approach to energy ablative therapy of small renal tumours: laparoscopic high-intensity focused ultrasound. European urology 2008; 53:810-816; discussion 817-818.

10. Han J, Kong Y, Xu Y, Tao Z, Wang Y. [Clinical observation and comparison on radiofrequency ablation and focused ultrasound in treatment of perennial allergic rhinitis]. Lin chuang er bi yan hou tou jing wai ke za zhi $=$ Journal of clinical otorhinolaryngology, head, and neck surgery 2011; 25:349-351.

11. Bousquet J, Khaltaev N, Cruz AAet al. Allergic Rhinitis and its Impact on Asthma (ARIA) 2008 update (in collaboration with the World Health Organization, GA(2)LEN and AllerGen). Allergy 2008; 63 Suppl 86:8-160.

12. Cheng LJ, Liu B, Ning B, Ming H, Wang C, Wan LX. High-intensity focused ultrasound for the treatment of allergic rhinitis using nasal endoscopy. Experimental and therapeutic medicine 2013; 5:320-322

13. Khokhlova VA, Bailey MR, Reed JA, Cunitz BW, Kaczkowski PJ, Crum LA. Effects of nonlinear propagation, cavitation, and boiling in lesion formation by high intensity focused ultrasound in a gel phantom. The Journal of the Acoustical Society of America 2006; 119:1834-1848.

14. Chen L, ter Haar G, Hill CR. Influence of ablated tissue on the formation of high-intensity focused ultrasound lesions. Ultrasound in medicine \& biology 1997; 23:921-931.

15. Tan G, Ma Y, Li H, Li W, Wang J. Long-term results of bilateral endoscopic vidian neurectomy in the management of moderate to severe persistent allergic rhinitis. Archives of otolaryngology--head \& neck surgery 2012; 138:492-497.

16. Unsal Tuna EE Ozbek $C, K o c C$ Ozdem C. Evaluation of nasal symptoms and mucociliary function in patients with allergic rhinitis treated with chemosurgery using trichloroacetic acid. American journal of otolaryngology 2008; 29:37-41.

17. Tsai YL, Su CC, Lee HS, Chen HC, Chen MK. Symptoms treatment for allergic rhinitis using diode laser: results after 6-year follow-up. Lasers in medical science 2009; 24:230-233.

18. Lin HC, Lin PW, Friedman Met al. Long-term results of radiofrequency turbinoplasty for allergic rhinitis refractory to medical therapy. Archives of otolaryngology--head \& neck surgery 2010; 136:892-895. 